\title{
Special issue on energy-aware computing and communications
}

\author{
Lizhe Wang • Samee U. Khan • Laurence T. Yang • \\ Feng Xia
}

Published online: 5 January 2013

(C) Springer Science+Business Media New York 2012

Nowadays, high-end computing facilities can consume a very large amount of power albeit they provide high performance computing solutions for scientific and engineering applications. Energy consumption for high-end facilities thus contributes to a significant electric bill. Additionally, high power consumption in general results in higher cooling costs. Furthermore, to allow computing facilities to operate on high power for a long time will lead to high temperature of computing systems, which further harms a system's reliability and availability. Therefore, reducing power consumption for high end computing becomes a critical research topic.

This special issue is devoted to the state-of-the-art research in the field of energy-aware high-end computing. A number of high quality research articles were submitted. We (the guest editors) had a very difficult decision to make on the inclusion and exclusion of research articles. Because of the space constraints, we could only include the following eight outstanding research articles:

L. Wang (ه)

Center for Earth Observation and Digital Earth, Chinese

Academy of Science, Beijing, P.R. China

e-mail: Lizhe.Wang@gmail.com

\section{S.U. Khan}

Department of Electrical and Computer Engineering, North

Dakota State University, Fargo, ND, USA

L.T. Yang

Department of Computer Science, St. Francis Xavier University, Antigonish, NS, Canada

F. Xia

School of Software, Dalian University of Technology, Dalian,

P.R. China
- An overview of energy efficiency techniques in cluster computing systems

- Classified power capping by network distribution trees for green computing

- Imbalance of CPU temperatures in a blade system and its impact for power consumption of fans

- Towards energy-efficient parallel analysis of neural signals

- CHERUB: power consumption aware cluster resource management

- DENS: data center energy-efficient network-aware scheduling

- Energy cost evaluation of parallel algorithms for multiprocessor systems

- Parallel application-level behavioral attributes for performance and energy management of high-performance computing systems

Guest Editors 